\title{
Extending AMCW Lidar Depth-Of-Field Using a Coded Aperture
}

\author{
John P. Godbaz ${ }^{1}$, Michael J. Cree and Adrian A. Dorrington \\ School of Engineering, University of Waikato, Hamilton, New Zealand
}

\begin{abstract}
By augmenting a high resolution full-field Amplitude Modulated Continuous Wave lidar system with a coded aperture, we show that depth-of-field can be extended using explicit, albeit blurred, range data to determine PSF scale. Because complex domain range-images contain explicit range information, the aperture design is unconstrained by the necessity for range determination by depth-from-defocus. The coded aperture design is shown to improve restoration quality over a circular aperture. A proof-of-concept algorithm using dynamic PSF determination and spatially variant Landweber iterations is developed and using an empirically sampled point spread function is shown to work in cases without serious multipath interference or high phase complexity.
\end{abstract}

\section{Introduction}

Full-field amplitude modulated continuous wave (AMCW) lidar systems utilise the time-of-flight (TOF) principle to generate two dimensional matrices of intensity and radial range values using active scene illumination. Whereas point and line scanner based systems require expensive mechanical systems to sequentially capture a point cloud, full-field systems capture an entire image simultaneously and near-instantly opening up a variety of applications including games, medical imaging, security and engineering quality control.

However, despite their advantages, full-field AMCW systems introduce new challenges such as systematic errors due to multipath interference and limited depth-of-field (DOF). In full-field AMCW lidar systems limited DOF results in both erroneous range and intensity values around the edges of objects as well as a loss of detail. While most previous computational photography work has addressed the DOF problem for intensity images using techniques such as coded apertures [1] and plenoptic cameras [2], previous systems have relied on implicit range information. Since AMCW lidar systems produce explicit range information, albeit limited by the DOF, there is inherently more information available to assist in restoration.

Prior depth-from-defocus (DFD) techniques $[3,4]$ utilise the known range variant properties of the PSF to determine distance, however typically require

\footnotetext{
${ }^{1}$ Contact email: jpg7@waikato.ac.nz
} 


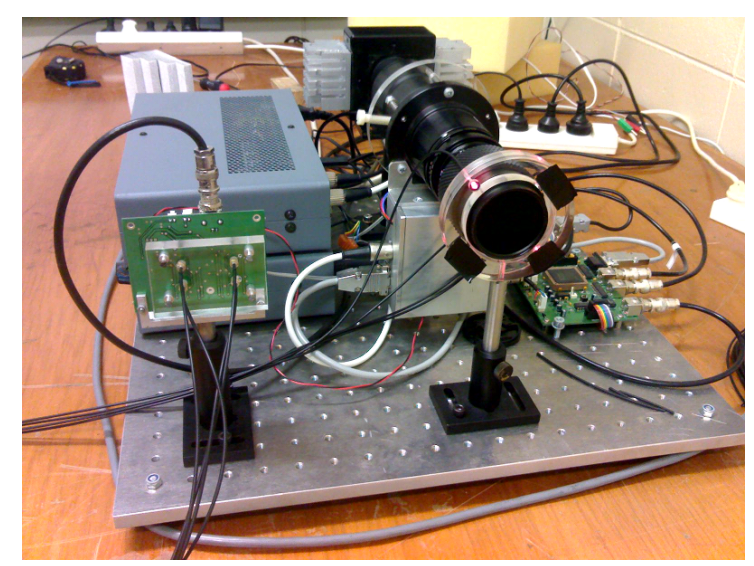

Fig. 1: Our full-field lidar system. While it may initially appear bulky and inelegant, it provides capabilities that existing commercial systems do not. In the configuration shown three of the four illumination sources are occluded. For this paper, all four illumination sources were utilised to provide coaxial illumination of the scenes.

more than one image of a scene. More modern methods have used coded apertures to make the blurring less of a low-pass filter and enable high quality restoration while requiring only a single image [1]. Related work has changed the nature of motion blur using coded fluttered shutter patterns [5]. Traditional plenoptic cameras allow refocussing without any explicit range information [2] however sacrifice spatial resolution. Alternative methods like Lumsdaine and Georgiev's 'Plenoptic 2.0' [6], which offer a substantial increase in resolution, require the determination of a range dependent magnification parameter in order to produce an artefact free image. Other techniques for defocus invariance include wavefront coding [7] and merging multiple images at different focal settings. Deconvolution techniques have been previously applied to full-field lidar images for the purposes of light scattering reduction [8,9]. Another work [10] blindly determined the focal parameters of a full-field lidar system and utilised them to improve DOF.

In this paper we briefly demonstrate the advantages of our coded-aperture design over a circular aperture for extending DOF and then show the deconvolution of real defocussed range-images captured using a coded-aperture variation of the full-field heterodyne AMCW lidar system from [11]. A picture of the system is given in fig. 1.

\section{Background Theory and System Design}

\subsection{AMCW Lidar}

AMCW lidar systems work by illuminating a target scene with modulated light and then sampling the correlation of the reflected light with a reference signal 
at the same or a slightly different frequency. The TOF results in a range variant phase shift in the returned illumination - this phase shift is typically measured by mixing the returned light with a reference signal using either a modulated CCD or CMOS sensor [12] or modulated image intensifier [11].

An image intensifier is typically used in devices like night vision goggles to amplify light intensity across a 2D field of view. By modulating the image intensifier gain at high frequency, it is possible to optically correlate the reference signal with the backscattered illumination modulation signal. A technique known as heterodyning allows the difficult, high frequency phase measurement problem to be reduced to an easier low frequency phase measurement problem. If the illumination modulation signal is at $x \mathrm{~Hz}$ and the reference modulation is at $y \mathrm{~Hz}$, then a downconverted correlation waveform is formed at $(x-y) \mathrm{Hz}$. Since the phase offset of the downconverted correlation waveform is proportional to that of the backscattered illumination signal, if $(x-y) \mathrm{Hz}$ is sufficiently low then phase can be calculated from data captured using an off-the-shelf CCD camera.

\subsection{The Range-Imager}

Fig. 2 shows the optical configuration of the ranger system. The scene is illuminated by modulated laser light and imaged by a Nikkor $50 \mathrm{~mm} \mathrm{f/1.8D} \mathrm{lens}$ where the aperture diaphragm blades are replaced with a coded aperture. The primary optics image the scene onto the mirror-like surface of the image intensifier photocathode. A phosphor screen displays the correlation of the returned scene illumination with the image intensifier modulation signal. This results in a temporally varying correlation waveform, where phase corresponds to object range. The phosphor screen is focussed onto a CCD image sensor using additional coupling optics, thus allowing the measurement of range and active intensity.

Raw range information is typically encoded as complex domain values and is generated by calculating the bin of the temporal discrete Fourier transform corresponding to the correlation waveform fundamental frequency for each pixel. This value corresponds to a sample of a particular bin of the spatial Fourier transform of component signal returns over range. For a single pixel composed of a single component return an ideal AMCW lidar measurement can be written as

$$
\eta=a \mathrm{e}^{4 \pi j d / \lambda}
$$

where $\eta \in \mathbb{C}$ is a complex domain range measurement, $a$ is the active intensity, $d$ is the distance from the camera and $\lambda$ is the illumination modulation wavelength.

In practice, AMCW lidar measurements are subject to systematic errors, particularly due to the impact of multipath interference. Multipath interference, of which mixed pixels are a type, is caused when a single pixel integrates light from sources at more than one range causing an erroneous range measurement the erroneous value being the sum of the complex domain range measurements of each component return. This can result in range-intensity coupling, where the measured range is a function of intensity. When a range image is subject to limited DOF, blurring of the edges of objects results in the formation of large 


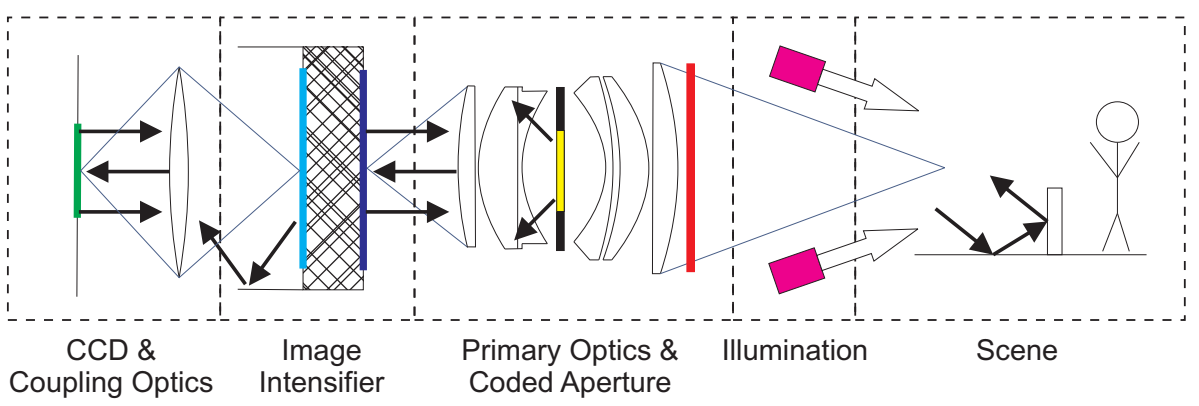

Fig. 2: The optical configuration of the range-imager. Key: modulated lasers (magenta), narrowband filter (red), coded aperture (yellow), image intensifier photocathode (blue), phosphor screen (cyan), CCD image sensor (green). Black arrows represent sources of multipath.

bands of mixed pixels containing erroneous values. One of the aims of this paper is to demonstrate that these erroneous values can be restored. Methods have been developed to mitigate [13] or find the component returns within mixed pixels [14, 15], however the output from these algorithms is difficult to incorporate into a simple deconvolution model. Since each component within a mixed pixel is at a different range from the camera, each has a different PSF. For this paper we model each pixel as being at a single discrete range, which while non-ideal, retains the simplicity of a single two dimensional image array.

At the moment full-field lidar image processing research is limited by the unavailability of off-the-shelf high resolution systems and the black-box nature of many commercial devices. The custom range-imager utilised for this paper has an effective resolution of around 200,000 pixels - many times that of any commercially available device. However, this comes at the cost of an increase in complexity due to the additional optics required to couple the image intensifier to the CCD and an increase in scattered light.

\subsection{Image Formation}

From geometric optics, the defocus PSF for an optical system is a scaled image of the aperture shape given by

$$
r_{p}=\alpha\left(1-\frac{\beta}{d}\right)
$$

where $r_{p}$ is the radius of the PSF, $d$ is the distance from the first principal plane to the object, $\beta$ is the distance from the first principal plane to the point on the optical axis at which objects are most in-focus and $\alpha$ is a scaling constant [10]. In the Fourier domain, convolution by a PSF corresponds to elementwise multiplication of the spatial frequencies of the image with the spatial frequencies of the PSF

$$
g=f \star h \Leftrightarrow G[u, v]=F[u, v] H[u, v]
$$


where $f$ is the original image, $g$ is the blurred image and $h$ is the PSF. Any spatial frequencies missing from the PSF are lost, making high quality image restoration difficult. A standard pillbox PSF is non-ideal because it has zeros in its MTF. A coded aperture works by inserting a device into the light path that changes the effective aperture, generally with the aim of improving the properties of the MTF. By whitening the MTF it is possible to improve the quality of restored images. Because there is explicit range information, it is possible to aim for as broadband a PSF as possible without the constraints imposed by extraction of implicit range information.

\section{$3 \quad$ Methodology}

\subsection{The Coded Aperture}

The coded aperture utilised for this paper is a $7 \times 7$ random noise pattern that was printed onto an overhead projector transparency (OHT) as shown in fig. 3. Due to the limited contrast provided by the printing process, the aperture pattern was augmented using marker pen - this resulted in slight unevenness, but had no other impact due to empirical sampling. Advantages of this method of aperture construction include low cost and that any pattern can be produced without physical constraints such as the connectivity required for a physical cut-out pattern. The biggest disadvantage is that depending on the type and quality of the OHT material, the aperture may contribute to light scattering and reflection within the ranger. Some previous approaches include cut out patterns [1] and LCD screens [16].

In order to compare our coded aperture design to a similarly sized circular aperture we simulated blurred and noisy intensity and phase images. Fig. 4 shows how the coded aperture improves the performance of deconvolution for an intensity image. For the Lena image at a SNR of $1000: 1$ there is a $24 \%$ decrease in RMS error in the restored image. Fig. 5 shows how the coded aperture affects the restoration of phase content in a pure phase image - that is a simulated range image where every pixel has a modulus of one, thus isolating the impact on phase information. The blurred phase information for the textured object counterintuitively appears to peak where there are troughs in the unblurred image due to the black regions in the centre of the aperture pattern. Despite designing the aperture for a white spectral response, limited Gibbs' phenomenon still occurs at hard discontinuities.

\subsection{The Point Spread Function}

The empirical point spread function of our system is formed as the convolution of the fixed point spread function of the image intensifier and CCD coupling optics with the range-variant point spread function of the primary optics. The image formation process for an AMCW range-imager is the same as for a standard camera with the exception that any reflections before the image intensifier result 


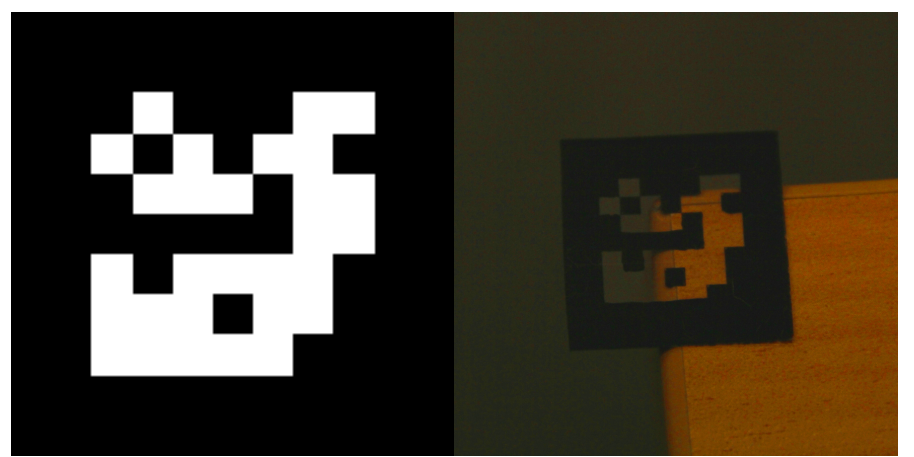

(a) Pattern

(b) Example OHT Aperture

Fig. 3: The binary pattern utilised for this paper and an example OHT based coded aperture.

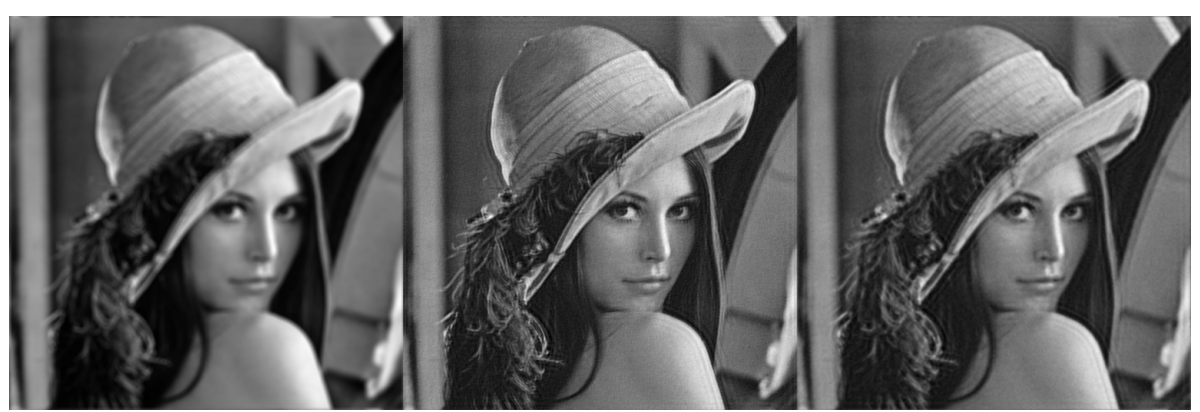

(a) Blurred Lena Image (b) Restoration (Coded)

(c) Restoration (Circular) (Circular)

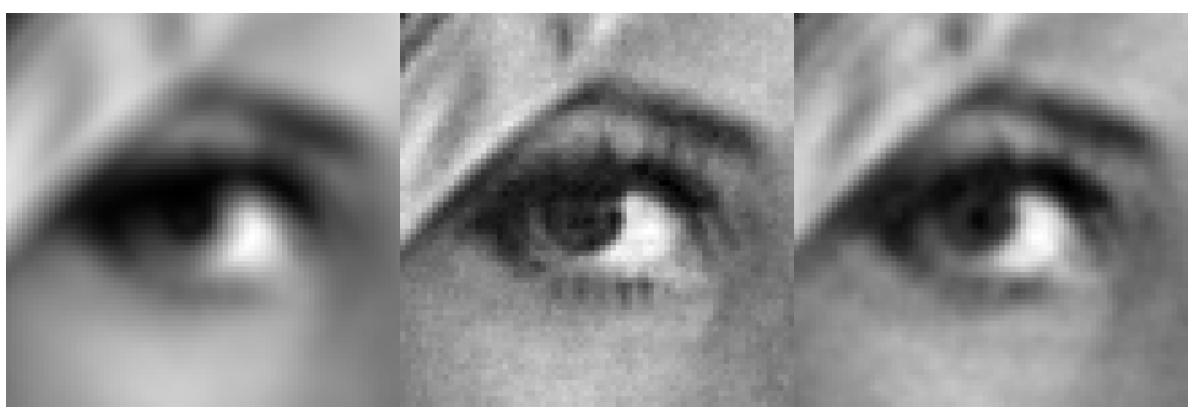

(d) Blurred Subregion (Cir-(e) Restored Subregion(f) Restored Subregion (Circular)

(Coded) cular)

Fig. 4: The impact of aperture choice on deconvolution restoration quality of an intensity image in the known, isoplanatic PSF case. Simulated at a SNR of $1000: 1, \lambda=0.015$ with 50 Landweber iterations. 


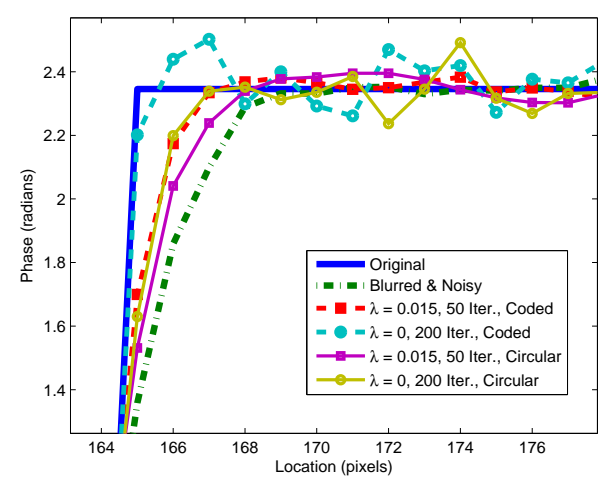

(a) Hard Discontinuity

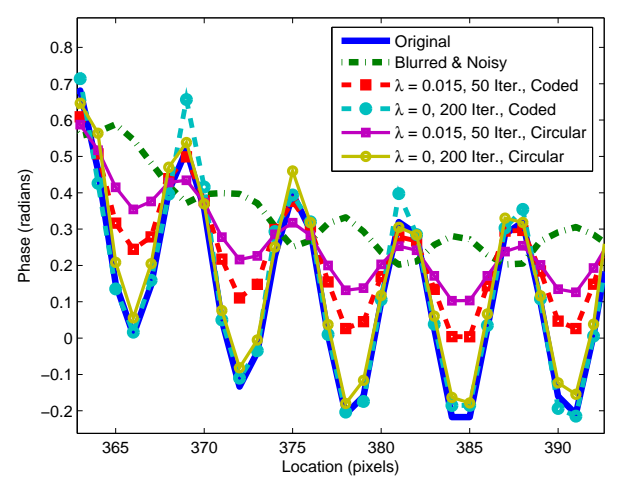

(b) Textured Object

Fig. 5: Slices through a simulated pure phase image pre- and post-deconvolution using a SNR of $1000: 1$. For a given regularisation constant the coded aperture generally results in better restoration quality than a circular aperture - the behaviour for the phase of a complex number is similar to that in the case of an intensity image, but with a slightly greater sensitivity to ringing.

in an increased TOF and thus a phase shift in the range measurements; fully modelling this requires the utilisation of a complex domain PSF.

Previous papers have sampled the PSF of a full-field AMCW lidar system both for the purpose of extending DOF [10] and for the purpose of mitigating multipath due to scattering in the range-imager optics $[8,9]$. While [9] utilised retroreflective dots, we utilise a fibre-optic based point source because it offers better performance while remaining subpixel in size. Attempting to measure both the defocus PSF and scattering effects at the same time is very difficult due to the extreme dynamic range required. In particular, temperature stability is extraordinarily important because even a slight change in bias can result in a massive redistribution of intensity from the defocus component of the PSF to the scattering component.

Fig. 6a shows how the PSF changes over range. Allowing for the image intensifier and coupling optics, the PSF scales in the manner predicted by eqn. 2. However the PSF samples close to the ranger are much more blurred than the PSFs of similar radius at a large distance - possible causes include optical abberations and scattering from the coded aperture. There is a slight pincushion effect on the PSF shape due to radial distortion from the component lenses.

The PSF also changes spatially; fig. $6 \mathrm{~b}$ shows the log intensity of the PSF in order to highlight subtle scattering effects. Most notably, there is an inverted image of the coded aperture present in the left-most image, which distorts and disappears as the point source is moved to the right side of the image - there is also a soft halo and some specular 'dots' (right-most image). Because of the 


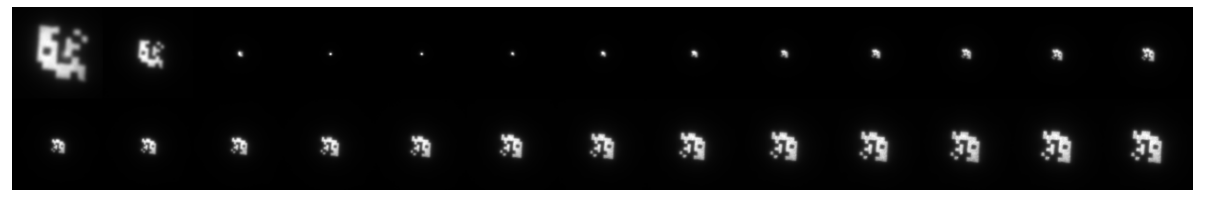

(a) PSF Range Variance (Intensity)

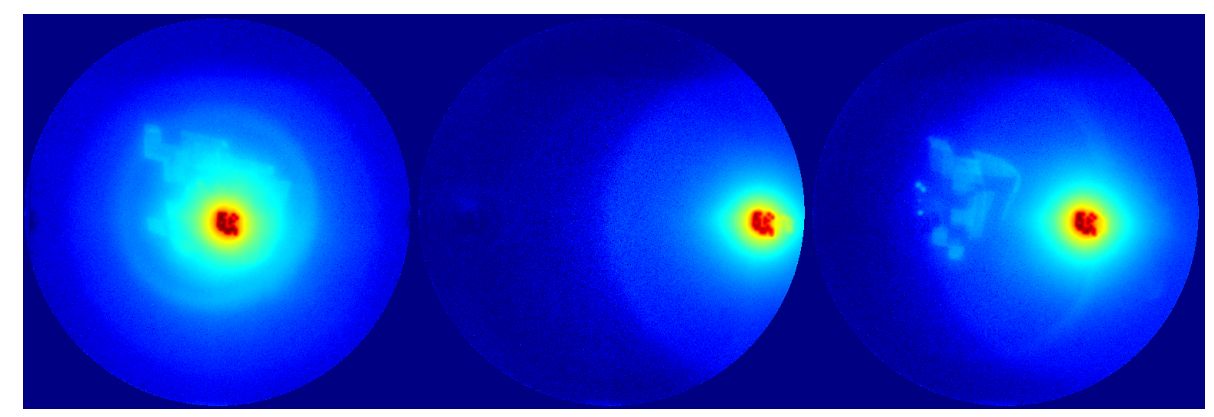

(b) PSF Spatial Variance (Log Intensity)
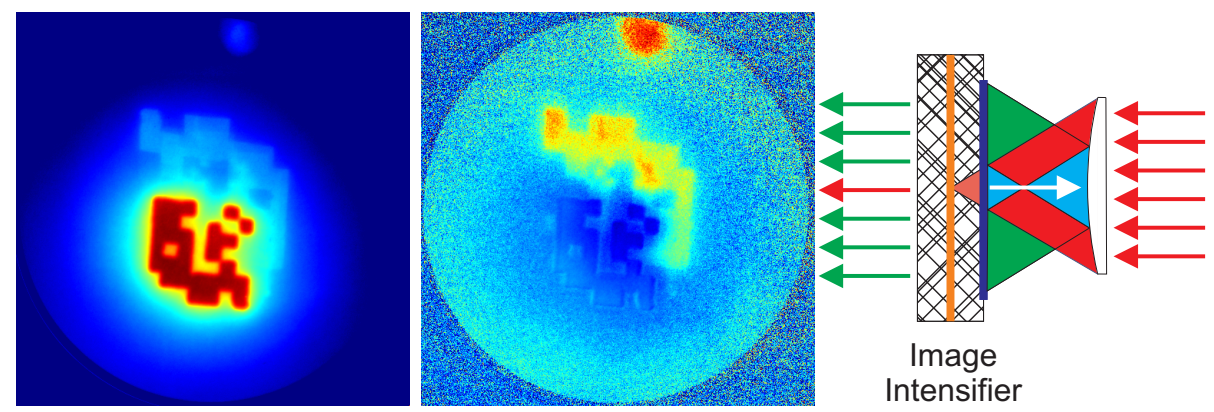

(c) Complex PSF - Log Intensity (left), Phase (right)

(d) PSF Formation Model

Fig. 6: Spatial and range variation in the coded-aperture range-imager PSF. In addition, the complex domain PSF is shown for the highly defocussed case showing subtle phase shifts in the scattered light. In log-intensity images, red represents high intensity and blue low. In phase images, red represents greater distance and blue less distance. From these data we can determine the formation process for the most prominent scattering. In fig. $6 \mathrm{~d}$, the initial aperture image (red) is reflected off the image intensifier and back to the final lens in the primary optics (cyan). Despite the low reflectivity of the lens, a significant amount of light is reflected back towards the image intensifier (green). The focal plane (orange) moves as the range to the point source changes, thus changing whether the primary PSF is inverted and the size of both the primary and reflected PSFs. The reflected PSF always has the same orientation. 
spatial complexity of the PSF, we only utilise centred PSF samples, otherwise the large number of PSF samples would greatly increase the computational complexity of the restoration.

Calculating the phase of extremely dark scattered light is very difficult, so barring inordinately long exposure times or image intensifier burn-in due to oversaturation it is only possible to image the complex domain PSF with extreme defocus. High levels of defocus allow the intensity of the scattered light to be increased while keeping the maximum image intensity to a safe region for the image intensifier. Thus while we still model scattering, we cannot plausibly model the slight phase shifts inherent in the scattering PSF across the entire PSF gamut. Fig. 6c shows the complex domain PSF for an extremely defocussed point source - note the low SNR for the darkest regions. Since the point source is within a few centimetres of the optics, the path length difference for light travelling through different sections of the aperture is visible - the path length varies by almost a centimetre within the primary/defocus PSF (blue/cyan). The reflections in the background have a much greater path length; the inverted aperture shape (yellow) has a path length at least $6 \mathrm{~cm}$ longer than the primary PSF and the reflection at the top (red) has a path length at least $7.5 \mathrm{~cm}$ longer. From this information, we can determine the formation process for the inverted image - this is given in fig. 6d. We are unaware of any previous measurements of the complex domain PSF of a full-field AMCW lidar system.

\subsection{Restoration Method}

We use a spatially variant Landweber [17] deconvolution method using a Gaussian spatial derivative prior and a weighting mask to remove boundary effects due to the image intensifier. By writing the spatially variant convolution as a matrix transformation, $f \star_{s v} h=T f$, each iteration becomes

$$
\hat{f}_{n+1}=\hat{f}_{n}+\gamma\left(T^{*} W\left(g-T \hat{f}_{n}\right)-\lambda L \hat{f}_{n}\right)
$$

where $\hat{f}_{n}$ is the estimate of the unblurred image at the $n$th iteration, ${ }^{*}$ is the Hermitian transpose of a matrix, $\gamma$ is a gain term, $W$ is a diagonal matrix of data weights, $\lambda$ is the regularisation parameter and $L$ is a Laplacian kernel. This is equivalent to iteratively minimising the function

$$
\epsilon(\hat{f})=\|W(g-T \hat{f})\|_{2}^{2}+\lambda\left\|D_{\mathrm{h}} \hat{f}\right\|_{2}^{2}+\lambda\left\|D_{\mathrm{v}} \hat{f}\right\|_{2}^{2}
$$

using gradient descent, where $D_{\mathrm{h}}$ is a horizontal derivative filter and $D_{\mathrm{v}}$ is a vertical derivative filter. The initial estimate is the captured blurred range-image. Additional blank, zero weighted boundaries are added to each image, increasing the image size from $512 \times 512$ to $768 \times 768$ to mitigate wraparound effects from the use of circular convolutions.

Before each iteration the PSF is dynamically determined for each pixel using radial distance calculated from the phase angle of value in $\hat{f}_{n}$. In general, distance along the optical axis can be approximated without calibration by the radial 


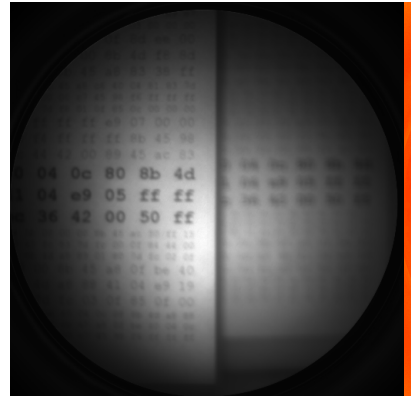

(a) Initial Modulus

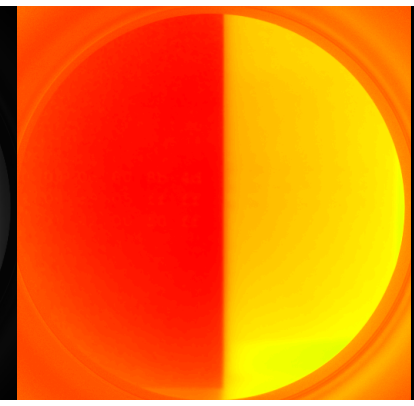

(b) Initial Phase

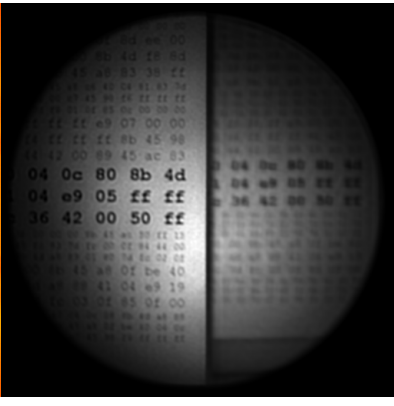

(c) Deconvolved Modulus

Fig. 7: Scene One, pre- and post-deconvolution. For the phase image, red represents objects closer to the camera (smaller phase offset) and yellow objects farther away (greater phase offset). The restoration of the hard phase discontinuity is shown in fig. 9a.

distance. A threshold is set for each restoration, usually 10 iterations, at which point the PSF stops being dynamically updated to prevent noise amplification. This method typically works quite well in regions with edge induced mixed pixels as the values tend to converge to a sharper edge, but in regions subject to severe range-intensity coupling due to scattered light the algorithm can fail.

\section{Results and Discussion}

Three different scenes were imaged of increasing spatial complexity: two boxes at varying distance from the ranger (fig. 7), a garden gnome and several patterned boards (fig. 8) and a chess set (fig. 10). Due to the optical configuration, ground truth was unavailable. Slices through the first two scenes are shown in fig. 9.

Scene one is an extremely simple scene containing two boxes printed with a test pattern. Fig. 7a shows the initial blurred modulus, which using the blurred range information from fig. $7 \mathrm{~b}$ is restored to the point where most of the text can be read - a substantial improvement in DOF. Fig. 9a shows how the phase is recovered during the deconvolution process - this graph shows a horizontal slice through the scene in the middle. The deconvolution process results in a substantial sharpening of the boundary between the two boxes as well as a significant shift in the range of the right hand box due to the partial removal of some scattered light. However there remains range-intensity coupling postdeconvolution most probably due to incomplete modelling of the spatial variance of scattering. It is extremely common in real images for range measurements to be shifted by $2-3 \mathrm{~cm}$ due to scattered light.

Scene two is a more complicated scene. Due to the larger dynamic range, the modulus images of both scenes two and three use gamma compression of $\gamma=0.5$. In this scene there is much more significant blurring and light scattering. Fig. 


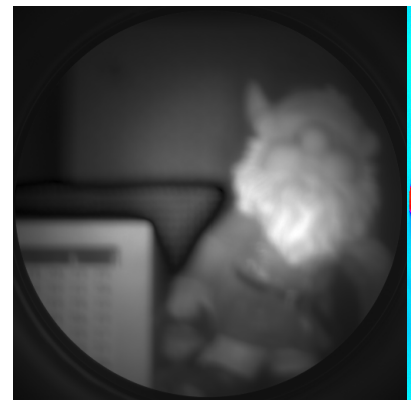

(a) Initial Modulus

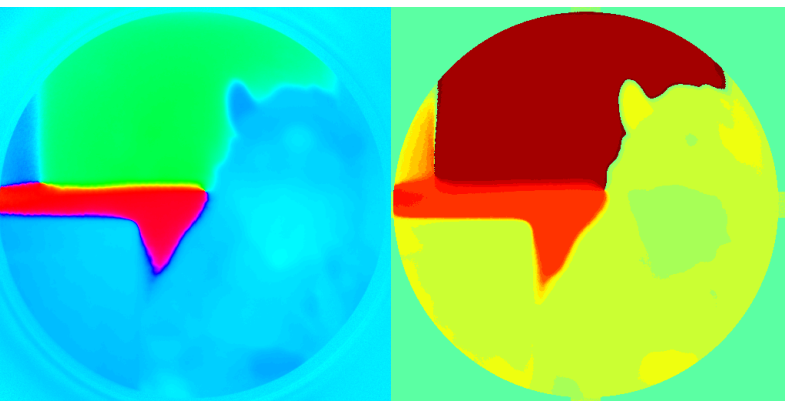

(b) Initial Phase (c) Initial PSF Number
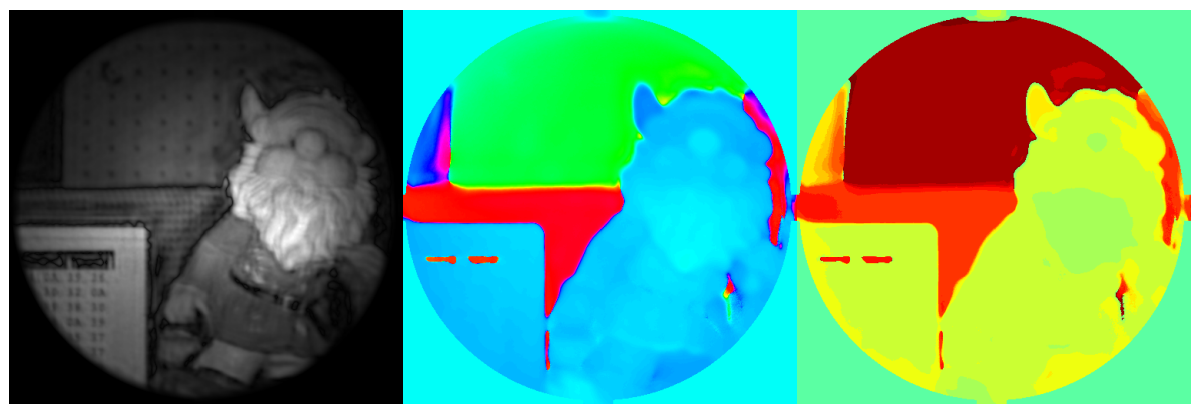

(d) Deconvolved Image(e) Deconvolved Modulus (200 Iter.) Phase (10 Iter.) Iter.)

Image(f) Final PSF Number (10

Fig. 8: Scene two, pre- and post-deconvolution. For the phase images, hue represents phase and is cyclic - in order of increasing phase: cyan, blue, magenta, red, yellow, green, cyan. Due to the high modulation frequency, the depth of the scene exceeds the ambiguity interval. While a large number of iterations increases modulus resolution substantially, it tends to introduce unnecessary ringing into phase information.

8c shows the initial PSF used for each pixel, by the 10th iteration the PSF has changed in regions such as between the garden gnome and front-most board (fig. 8f). In the final deconvolved range-image the modulus (fig. 8d) and phase (fig. $8 \mathrm{e})$ components have substantially improved sharpness, although there are some notable artefacts. Most noticeable is the erroneous range value given for the black tape holding the test pattern onto the front board - the red range value is roughly equivalent to phase shifting the correct range value by $\pi$ radians and this may indicate excessive compensation for scattering. There are ringing effects around the edges of objects such as the head of the gnome and the pattern. Like many real-life range-images, scene two contains a small region at the top left which is outside the range ambiguity interval - ie. due to the modulo $2 \pi$ nature of phase, this region has been deconvolved by an incorrect PSF. This is unavoidable for 


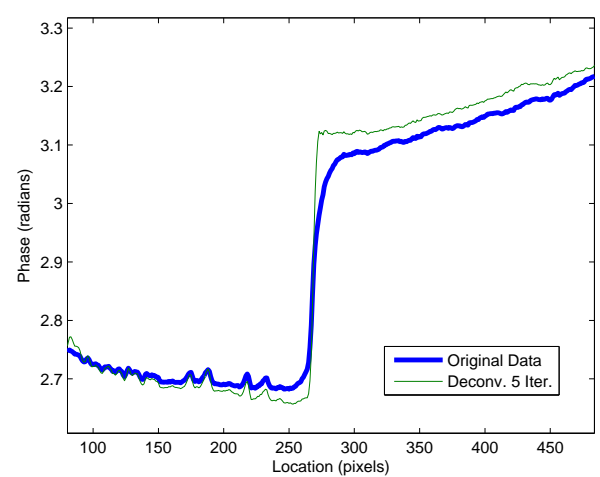

(a) Scene One

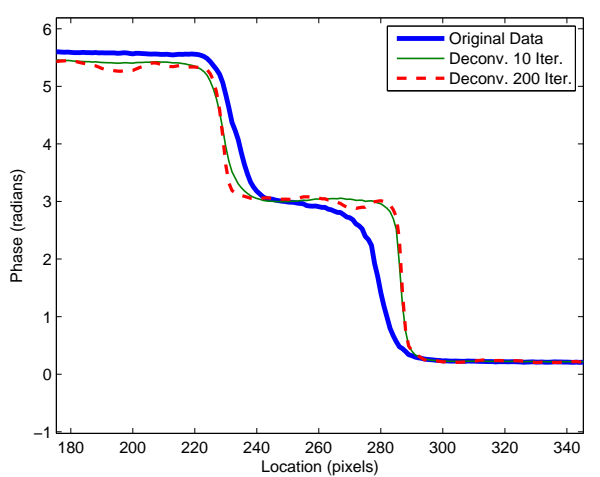

(b) Scene Two

Fig. 9: The phase of slices through scenes 1 and 2, before and after deconvolution. From a phase perspective, 200 iterations provides few benefits over 10 iterations. Fig. 9a shows range-intensity coupling before and after restoration.

real-world scenes unless range precision is sacrificed by using a particularly low modulation frequency or a phase unwrapping method utilised.

Unlike normal intensity images, complex domain range-images have some complicated behaviour around edges. In typical scenes the edges of objects are mixed pixels, however these tend to be heavily attenuated by the deconvolution process, resulting in dark bands at the boundaries of objects. A different type of dark band is seen in defocussed images where the objects have sufficiently different phases as to result in partial cancellation - these bands can be seen around the edges of the chess pieces in fig. 10a. While a smoothness constraint may limit the impact of noise on the restoration, it also has a tendency to intensify dark bands between objects at significantly different ranges. If the aim of a restoration is to produce an in-focus pure intensity type image, then it may be more appropriate to deconvolve the total integrated intensity, which is essentially the total amount of light detected by the ranger. Albeit, most commercial ranger-imagers use a differential measurement process that removes this information from the raw measurements.

Scene three demonstrates the current limitations of the restoration algorithm. The extreme range-intensity coupling is demonstrated by the black chess pieces. Regions such as the knight's head, which is near black, are perturbed by light scattered from the board in the background resulting in PSF misestimation in addition to having very complicated range content. Since none of the image is saturated, the regions with specular reflections have the most accurate range measurements, which are visibly different from adjacent areas. This is compounded by the fact that each component at a different range within a pixel has a different PSF. Successful restoration of this type of scene awaits a more 


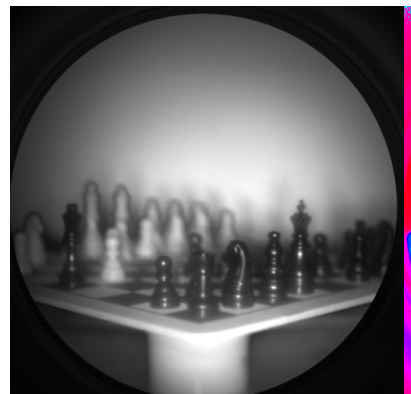

(a) Initial Modulus

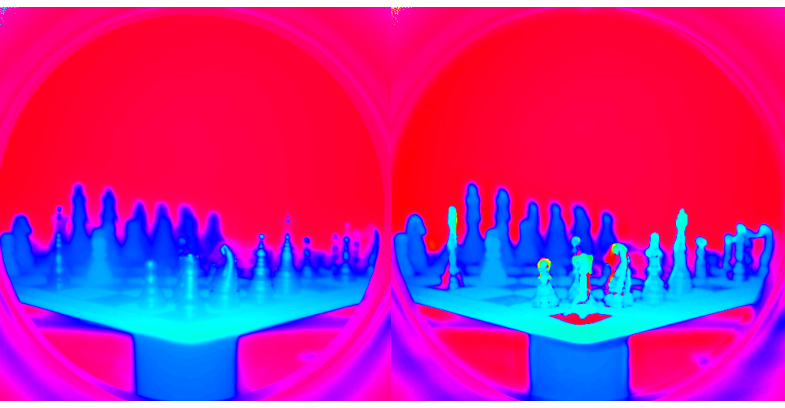

(b) Initial Phase (c) Failed Deconvolution (10 Iter.)

Fig. 10: Scene three, pre- and post-deconvolution. For the phase images, cyan represents objects closer to the camera and red objects farther away. This scene suffers from severe multipath contamination, as shown by the range-intensity coupling for the black chess pieces and squares. A combination of multipath and high phase complexity results in an unsuccessful deconvolution.

advanced restoration algorithm that takes into account the range of possible components within each pixel rather than making a naïve assumption that each sample is of an unperturbed single component return.

\section{Conclusion}

In this paper we have designed a broadband coded-aperture for coding defocus so as to allow depth-of-field to be extended through deconvolution. We have demonstrated that the coded aperture design results in an improvement in restoration performance over a circular aperture and incorporated the coded aperture design into a real full-field AMCW lidar system. The range variation of the defocus and scattering PSFs was sampled and reflection off the image intensifier was isolated as a significant contributor to scattered light. A naïve, proof-of-concept restoration algorithm was demonstrated to substantially improve the quality of some, but not all range-images captured using this new system - difficulties including misestimation of the restoration PSF due to multipath and the naïve assumption of a single component return.

Acknowledgement. This research was supported by a Tertiary Education Commission Top Achiever Doctoral Scholarship and the University of Waikato Strategic Investment Fund.

\section{References}

1. Levin, A., Fergus, R., Durand, F., Freeman, W.T.: Image and depth from a conventional camera with a coded aperture. In: Proc. SIGGRAPH 07. (2007) 
2. Ng, R., Levoy, M., Bredif, M., Duval, G., Horowitz, M., Hanrahan, P.: Light field photography with a hand-held plenoptic camera. Technical Report CTSR 2005-02, April 2005, Stanford University (2005)

3. Pentland, A.P.: A new sense for depth of field. IEEE Trans. Pattern Anal. Mach. Intell. 9 (1987) 523-531

4. Chaudhuri, S., Rajagopalan, A.N.: Depth from defocus: a real aperture imaging approach. Springer Verlag (1999)

5. Raskar, R. Agrawal A. Tumblin, J.: Coded exposure photography: motion deblurring using fluttered shutter. ACM Trans. Graph 25 (2006) 795-804

6. Lumsdaine, A. Georgiev, T.: Full resolution lightfield rendering. Technical report, Adobe Systems Inc. (2008)

7. Dowski, E.R., Johnson, G.E.: Wavefront coding: a modern method of achieving high-performance and/or low-cost imaging systems. In: Society of Photo-Optical Instrumentation Engineers (SPIE) Conference Series. Volume 3779. (1999) 137-145

8. Mure-Dubois, J., Hugli, H.: Optimized scattering compensation for time-of-flight camera. In: Two- and Three-Dimensional Methods for Inspection and Metrology V. Volume 6762., Boston, MA, USA, SPIE (2007)

9. Kavli, T., Kirkhus, T., Thielemann, J.T., Jagielski, B.: Modelling and compensating measurement errors caused by scattering in time-of-flight cameras. In Huang, P.S., Yoshizawa, T., Harding, K.G., eds.: Two- and Three-Dimensional Methods for Inspection and Metrology VI. Volume 7066., SPIE (2008) 706604

10. Godbaz, J.P., Cree, M.J., Dorrington, A.A.: Blind deconvolution of depth-of-field limited full-field lidar data by determination of focal parameters. In: Computational Imaging VIII, Proc. SPIE vol. 7533, San Jose, California (2010)

11. Dorrington, A.A., Cree, M.J., Payne, A.D., Conroy, R.M., Carnegie, D.A.: Achieving sub-millimetre precision with a solid-state full-field heterodyning range imaging camera. Meas. Sci. and Tech. 18 (2007) 2809-2816

12. Oggier, T., Lehmann, M., Kaufmann, R., Schweizer, M., Richter, M., Metzler, P., Lang, G., Lustenberger, F., Blanc, N.: An all-solid-state optical range camera for $3 \mathrm{D}$ real-time imaging with sub-centimeter depth resolution (SwissRanger). In: Optical Design and Engineering. Volume 5249., St. Etienne, France, SPIE (2004) $534-545$

13. Larkins, R.L., Cree, M.J., Dorrington, A.A., Godbaz, J.P.: Surface projection for mixed pixel correction. In: Image and Vision Computing New Zealand (IVCNZ'09), Wellington New Zealand (2009)

14. Godbaz, J.P., Cree, M.J., Dorrington, A.A.: Multiple return separation for a fullfield ranger via continuous waveform modelling. In: Image Processing: Machine Vision Applications II, Proc. SPIE vol. 7251, San Jose, California (2009)

15. Godbaz, J.P., Cree, M.J., Dorrington, A.A.: Mixed pixel return separation for a full-field ranger. In: Proc. IVCNZ 2008, Christchurch, New Zealand (2008)

16. Marcia, R.F., Harmany, Z.T., Willett, R.M.: Compressive coded apertures for highresolution imaging. In: Optics, Photonics, and Digital Technologies for Multimedia Applications. Volume 7723., SPIE (2010) 772304

17. Landweber, L.: An iteration formula for Fredholm integral equations of the first kind. Am. J. of Math. 73 (1951) 615-624 